\title{
New frontiers in rehabilitation. Oxygen-ozone therapy and neuronal plasticity in the treatment of Parkinson's symptoms
}

\author{
Luca Morelli, ${ }^{1-3}$ Simona Carla Bramani, ${ }^{1}$ Alessandra Guarino ${ }^{1,2}$ \\ ${ }^{1}$ San Fedele Multi-specialist Rehabilitation Centre, Longone al Segrino, Como, Italy; ${ }^{2}$ UCM United Campus of Malta, \\ Smart City, Lugano, Switzerland; ${ }^{3}$ Scientific Society of Oxygen-Ozone Therapy (SIOOT), Gorle (BG), Italy
}

\begin{abstract}
Oxygen-ozone therapy is effective for both activation of the cerebral microcirculation and increasing energy production by neurons, as recent studies have shown. As well as this, it has been shown to have a proven anti-inflammatory and antispasmodic action, improve microcirculation, increase the production of cellular energy, activate the mitochondrial antioxidant system and facilitate the elimination of cell catabolites. It also seems that oxygen-ozone therapy improves and prolong the effects of drugs such as Levodopa, Selegiline and Bromocriptine and antioxidants, therefore these activities could help us understand the improvements recorded in Parkinson's patients treated with oxygen-ozone therapy. The aim of our work was to carry out a postural, stabilometric and symptomatological assessment of the effects of oxygen-ozone therapy by using rectal insufflation in patients who undertook the intense motor activity of non-contact boxing. The results showed a significant reduction in the myofunctional, postural and stabilometric symptoms of Parkinsonian patients who practiced non-contact boxing activities, implemented though systemic oxygen-ozone therapy with insufflation.
\end{abstract}

\footnotetext{
Correspondence: Luca Morelli, Scientific Society of Oxygen-Ozone Therapy (SIOOT), via Don Luigi Sturzo 2, 24020 Gorle (BG), Italy. Tel:. 035.19910105.

E-mail: info@ossigenoozono.it

Key words: neuronal plasticity; Parkinson's symptoms; oxygen-ozone therapy; ozone therapy; rock steady boxing.

Contributions: the authors contributed equally.

Conflict of interest: the authors declare no potential conflict of interest.

Received for publication: 20 April 2018.

Accepted for publication: 21 April 2018.

(C) Copyright L. Morelli et al., 2018

Licensee PAGEPress, Italy

Ozone Therapy 2018; 3:7511

doi:10.4081/ozone.2018.7511

This article is distributed under the terms of the Creative Commons Attribution Noncommercial License (by-nc 4.0) which permits any noncommercial use, distribution, and reproduction in any medium, provided the original author(s) and source are credited.
}

\section{Introduction}

Parkinson's disease is a progressive neurological disease, which is linked to neuronal degeneration, and classified as a form of movement disorder.

Rehabilitation treatment for patients with Parkinson's disease is based on several principles aimed at improving respiratory and postural abilities through exercises to rebalance the tendency towards trunk flexion (Camptocormia); to improve coordination, muscle and joint function, reduce the disabling postural blocking of starting or continuing movement (Freezing), swallowing, walking, proprioception and posture.

The efficacy of adapted motor activities in patients with Parkinson's disease onthe symptoms and complications linked to the motor problems of those affected is well known in the literature. ${ }^{1-10}$

As part of this rehabilitative protocol, we have added the Rock Steady Boxing method, which is based on the efficacy of the nervous systems neuronal plasticity when responding to intrinsic and extrinsic stimuli, reshaping brain structures, functions and synaptic connections which is induced by reflex motor activity which is probably determined by the limbic system and the modulating action of the amygdala gland, stimulated by non-contact boxing, in escape and attack reactions. This interesting therapeutic opportunity for the symptoms of Parkinson's exploits the physiological plasticity of the human brain, with its continuous reorganisation of neuronal networks and creation of new brain circuits, which is induced above all by intense aerobic exercise in the presence of oxygen.

Oxygen-ozone therapy is effective for both activating the cerebral micro-circulation and increasing energy production by neurons, as recent studies have shown, in addition to which it has been shown to have a demonstrable anti-inflammatory and antispasmodic action, to improve the micro circulation, increase production of cellular energy, activate the mitochondrial antioxidant system and facilitate the elimination of cell catabolites. It also appears that oxygen-ozone therapy improves and prolongs the effects of drugs such as Levodopa, Selegiline and Bromocriptine and antioxidants. As a consequence, these activities could help us understand the improvements observed in Parkinson's patients treated with oxygen-ozone therapy.

The aim of our work was to carry out a postural, stabilometric and symptomatological assessment of the effects of oxygen-ozone therapy by using rectal insufflation in patients who undertook the intense motor activity of non-contact boxing.

\section{Materials and Methods}

As shown in Table 1, of our 14 heterogeneous patients who practiced no-contact boxing activities on a regular basis, with a 
diagnosis varying from stage 1 to stage 4 Parkinson's disease on the Hoehn and Yahr scale, which is useful for defining the patient's clinical stage, only 6 patients agreed to participate in our observational study.

The therapeutic oxygen-ozone treatment protocol we adopted in this study was treatment by rectal insufflation. The choice of this method vs Major Autohaemotherapy (GAET) was authorised by our Ethics Committee for its low invasiveness and criticality, particularly in the placebo phase of treatment. The study was conducted double-blind; both the patients and operators were unaware of the mixture that was insufflated.

Parkinsonian patients, who performed regular Rock Steady Boxing activities, twice a week, were treated biweekly for 10 weeks with a therapeutic mixture of oxygen-ozone, and a placebo mixture of air, in two different periods.

For treating Parkinson's disease with oxygen-ozone therapy, the specific protocol of the Italian Scientific Society of Oxygen Ozone Therapy (SIOOT) was adopted.

Random patients were divided into 2 groups of 3 patients, called Group A and Group B. Group A underwent rectal insufflation therapy with $150 \mathrm{cc}$ of oxygen-ozone at a concentration of 35 micrograms $/ \mathrm{ml}$ twice a week for the first 5 weeks. In the following 5 weeks, Group A was treated with a biweekly rectal insufflation placebo of air.

Group B was treated with placebo rectal insufflation therapy with $150 \mathrm{cc}$ of air biweekly for the first 5 weeks. In the following 5 weeks, Group B was treated with rectal insufflation therapy using $150 \mathrm{cc}$ of oxygen-ozone at a concentration of 35 micrograms $/ \mathrm{ml}$ twice a week.

In the Baseline (T0) assessments, after the 10th insufflation session (T1) and after the 20th insufflative session (T2), all patients underwent a psychometric assessment using the Fullerton Advanced Balance Scale, aimed at identifying active subjects with Parkinson's disease who are at risk of injuries from falls because of sensory and postural control problems. The test involved 10 activities based on performing both dynamic and static balancing in various situations, to identify the balance deficit of our patients, who were given a score from 0 to 4 for each activity performed, up to a maximum total of 40 points.

The Cut-Off score, with a high risk of falls, was considered to be less than or equal to $25 / 40$.

Patients also underwent the following instrumental postural assessments: Spinometric, using video rastersteriography with Diers Formetric 4D from the Hakomed company, and a BaropodometricStabilometricevaluation using a static and dynamic Pedoscan Professional static and dynamic Diers from the company Hakomed, for Baseline assesments (T0), after the 10th insufflation session (T1) and after the 20th insufflation session (T2).

Table 1. Heterogeneous patients who practiced no-contact boxing activities on a regular basis.

\section{Hoehn and Yahr Scale}

Stage 1: Unilateral disease.

Stage 2: Bilateral disease without balance involvement.

Stage 3: mild to moderate disease, some independent postural instability.

Stage 4: Established disease, still able to ambulate independently

Stage 5: Patient wheelchair bound or bedridden

\section{Results and Discussion}

Data from the patients examined in our observational study did not provide any statistically significant data, but several interesting observations were highlighted.

All of the patients showed improvements in symptoms associated with neuronal neoplastic activity, induced by non-contact boxing, insufflation treatment with ozone oxygen therapy as well as a greater stability and reduction in the risk of falls, as can be seen from the scores obtained in the Fullerton Advanced Balance Scale evaluation, shown in Figure 1. Further confirmation of greater stability was shown using the postural spinometric examination, with the reduction or stabilisation of the Camptocormic symptom given on extending the trunk of the Parkinsonian patient, as shown in Figure 2. Further evidence was shown by the reduction in anteroposterior flexion, determined by projection of the prominent vertebra (VP) to the medio-sacral (DM) (Figure 3) towards normal values and the increased harmonisation of the kyphotic dorsal and lordotic lumbar angles (Figures 4 and 5).

\section{Conclusions}

The results showed a significant reduction in myofunctional, postural and stabilometric symptoms in Parkinson's patients who practised non-contact boxing activities, which were implemented under systemic oxygen-ozone therapy with insufflation. It was

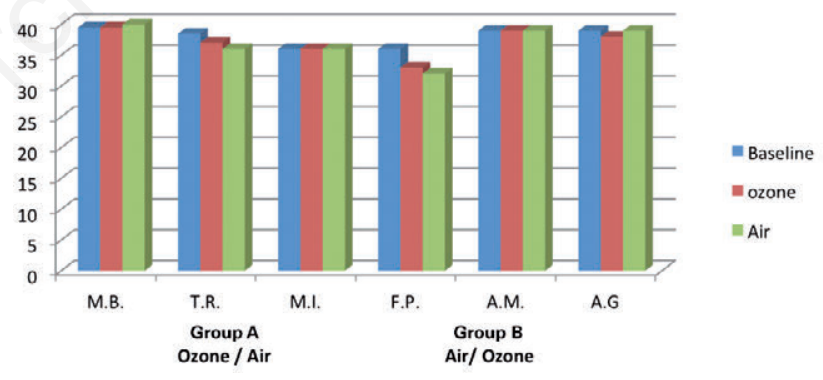

Figure 1. Scores obtained in the Fullerton Advanced Balance Scale evaluation.

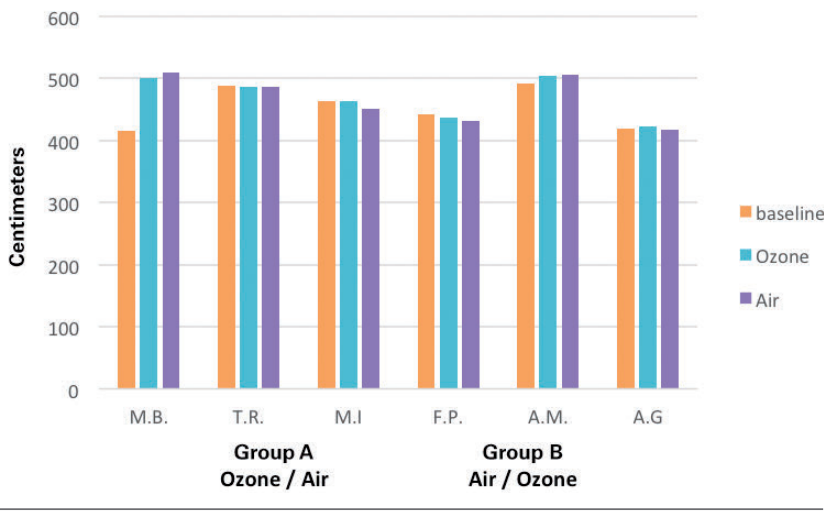

Figure 2. Reduction or stabilisation of the Camptocormic symptom given on extending the trunk of the Parkinsonian patient. 


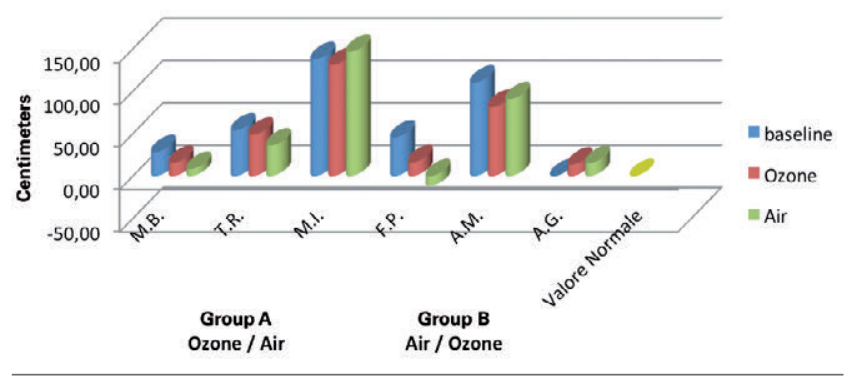

Figure 3. Antero- posterior flexion, determined by projection of the prominent vertebra to the medio-sacral.

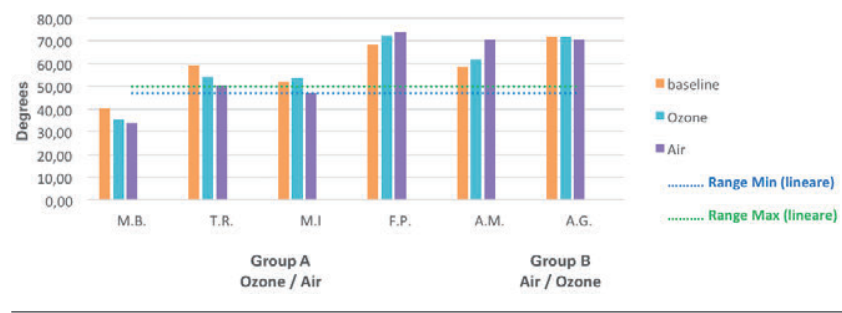

Figure 4. Kyphotic dorsal angles.

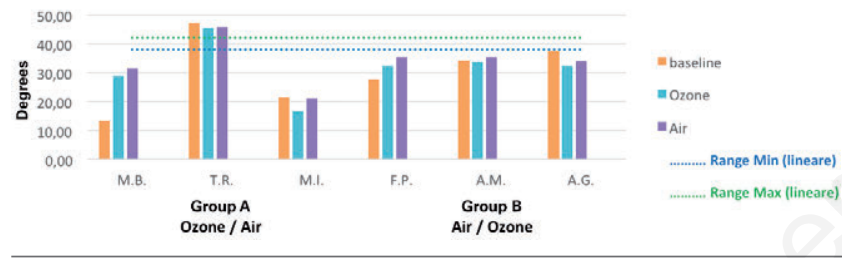

Figure 5. Lordotic lumbar angles.

interesting to note that patients in Group A showed, some albeit minimal, benefit from placebo insufflation therapy with air; this observation could be due to persistence of hypertoxic actions in the brain induced by the initial oxygen-ozone therapy.

The aim of our work and our future objectives are to offer Parkinson's patients an interesting therapeutic opportunity, which exploits both the human brain's physiological plasticity, which is induced by non-contact boxing in a rehabilitative and motor sports setting, and the chronic hypertoxic efficacy of systemic oxygenozone therapy on the cerebral micro-circulation and the greater energy production of neurons, in the reduction of the Parkinson's Symptoms.

\section{References}

1. Morelli L, Bramani SC, Galletta A, et al. Un pugno al Parkinson: Terapia dei sintomi del Parkinson tra sport e riabilitazione. Esperienza Italiana della Rock Steady Boxing Como Lake. Med Sport 2016;69:534.

2. Molinari F, Simonetti V, Franzini M, et al. L'autoemoterapia ozonizzata induce cambiamenti metabolici cerebrali a lungo termine nei pazienti affetti da sclerosi multipla. J Immunopathol Pharmacol 2014;379-89.

3. Combs SA, Diehl MD, Staples WH, et al. Boxing training for patients with Parkinson's disease: a case series. Phys Ther 2011;91:132-42.

4. Hirsch MA, Farley BG. Exercise and neuroplasticity in persons living with Parkinson's disease. Eur J Phys Rehabil 2009; 45:215-28.

5. Ahlskog JE. Does vigorous exercise have a neuroprotective effect in Parkinson disease? Am Acad Neurol 2011;77:288-94.

6. Ridgel AL, Vitek JL, Alberts JL. Not voluntary, exercise improves motor function in Parkinson's disease patients. Neurorehabil Neural Repair 2009;23:600-8.

7. Schlenstedt C, Brombacher S, Hartwigsen G, et al. Comparing the Fullerton Advanced Balance Scale with the mini-BESTest and Berg Balance Scale to assess postural control in patients with Parkinson disease. Arch Phys Med Rehabil 2014;96:218-25.

8. Fusco C, Zaina F, Negrini S. Colonna vertebrale: utilità delle nuove tecnologie per la valutazione. Il Fisioterapista 2010;1:27-33.

9. Mangone M. La spinometria Formetric nella valutazione non invasiva delle deformità del rachide e del tronco. Università La Sapienza, Roma; HAKOMED Italia-Biomedical Products, Congresso 8-9 maggio 2010.

10. Kluba T, Schäfer J, Hahnfeldt T, Niemeyer T. Prospective randomized comparison of radiation exposure from full spine radiographs obtained in three different techniques. Eur Spine J 2006;15:752-6. 